\title{
Analytic solution of nonlinear batch reaction kinetics equations
}

\author{
S. S. Motsa ${ }^{1}$ S. Shateyi ${ }^{2}$
}

(Received 31 July 2009; revised 5 March 2010)

\begin{abstract}
The classical nonlinear reaction kinetics equations are solved using an analytic technique for solving nonlinear problems known as the homotopy analysis method. An explicit analytic solution for the concentration of reactants and products that is uniformly valid for all times is presented. Numerical simulations based on Runge-Kutta initial value problem solvers verify our analytic solutions with good agreement.
\end{abstract}

\section{Contents}

1 Introduction

E38

2 Mathematical formulation

E41

3 Homotopy analysis method solution

E42

3.1 Explicit series solution of the batch reaction kinetics equations

E46

http://anziamj . austms.org.au/ojs/index.php/ANZIAMJ/article/view/2250 gives this article, (c) Austral. Mathematical Soc. 2010. Published March 17, 2010. ISSN 1446-8735. (Print two pages per sheet of paper.) 


\section{Introduction}

Batch reactors provide flexible means of producing high value-added products in specialty chemical, bio-technical, and pharmaceutical industries. To realize the production objectives, these batch reactors have to be operated optimally in a precise fashion. Batch reactor design has been studied from various perspective in order to develop systematic optimization tools to improve performance [1]. Friedrich and Perne [2] presented that the design of batch reactors means not only the design of equipment, but also the design of operation.

In the last four decades or so, many chemical producers moved from the relatively stable world of large continuous plant production to the much more turbulent environment of multi-product batch production in order to better adjust to changing market conditions. Kawarasaki et al. [3] intensively investigated and optimized several reaction conditions of cell-free protein synthesis such as temperature, buffers, tRNAS, and creatine phosphate.

Fulcher et al. [4] investigated and then provided insight into, the physical behaviour of the reaction mixture, and to evaluate the effectiveness of intrusive nutrient addition. The study showed that results for unstirred, batch zeolite A reaction systems the non-solid gel-fraction changed during the course of the reaction. Bonvin [5] presented a personal, thus necessarily subjective, view of the operation of batch and semi-batch reactors. The emphasis in this review was on the analysis of industrial challenges and the definition of academic opportunities. 
Van Woezik and Westerterp [6] focused on the thermal dynamics of a semibatch reactor, in which multiple exorthermic, liquid-liquid reactions are carried out. Muske et al. [7] presented a comparison of results obtained from deterministic and stochastic model-based optimization approaches for the determination of the optimal open-loop operating policy for a semi-batch reaction system. Hua et al. [8] proposed a cascade closed-loop optimization and control strategy for batch reactors.

Zhang and Smith [9] addressed a systematic methodology for batch and semibatch reactor design and optimization for ideal and non-ideal mixing. The method starts from a general representation in the form of a temporal superstructure based on the similarity of between plug flow reactors and ideal batch reactors. Goncalves et al. [10] compared the performance of batch and semi-batch reactors, under optimal operational conditions of amoxicillin enzymic synthesis at $25^{\circ}$ and $\mathrm{pH} \approx 6.5$. Most recently, Jana and Adari [11] dealt with the advanced adaptive control of a batch reactive distillation column for the production of ethyle.

Few, if any, attempted to solve batch problems analytically. To that end, this paper aims to obtain an analytic solution of these nonlinear batch reaction kinetics equations by using the homotopy analysis method (HAM). This is a fairly new technique that has been successfully applied in the analysis of systems of nonlinear equations in other areas of science and engineering particularly in fluid dynamics. Liao [12] gave a systematic description of homotopy analysis method (HAM), by means of an operator to denote non-linear differential equations in general. They also generally discussed the convergence of the related approximate solution sequences and showed that, as long as the approximate solution sequence given by the HAM is convergent, it must converge to one solution of the non-linear problem under consideration. Liao [13] further improved the homotopy analysis method and systematically described it through a typical non-linear problem.

Liao [14] applied the HAM to give a convergent series solution of non-similarity boundary-layer flows. Mehmood and Ali [16] investigated the incompressible 
generalized viscous flow with heat transfer analysis in the presence of viscous dissipation. Complete analytic solutions for velocity and temperature were obtained by homotopy analysis method. Ali and Mehmood [17] dealt with the unsteady boundary layer flow of viscous fluid in porous medium started due to the impulsively stretching of the plane wall using the homotopy analysis method. Mehmood et al. [18] presented a complete analytic solution to the unsteady heat transfer flow of an incompressible viscous fluid over a permeable plane wall. The homotopy analysis method was also used by Hayat et al. [19] to investigate the flow of a fourth grade fluid past a porous plate. Sajid and Hayat [20] proved that the perturbation and homotopy perturbation solutions for two problems, (i) unsteady convective-radiative equation and (ii) non-linear convective-radiative conduction equation, are only valid for weak non-linearity.

Cheng et al. [21] presented a similar solution for the nano boundary layer with a Navier boundary condition. The work considered three types of flow: (i) flow past a wedge; (ii) flow in a convergent channel; (iii) flow driven by an exponentially varying outer flow. The resulting differential equations are solved by the homotopy analysis method. Alizadeh-Pahlavan and Sadegy [22] studied unsteady MHD flow of a Maxwellian fluid above an impulsively stretched sheet, under the assumption that boundary layer approximation is applicable. Bararnia et al. [23] employed the homotopy analysis method to investigate the momentum, heat and mass transfer characteristics of MHD natural convection flow and heat generation fluid driven by a continuously moving permeable surface immersed in fluid saturated porous medium. Khan et al. [24] applied the homotopy analysis to develop an analytic approach for nonlinear differential equations with time delay. Allan [25] used the HAM to solve a non-linear, chaotic system of ordinary differential equations (Lorenz system). Xu et al. [26] investigated the time fractional partial differential equations by means of the homotopy analysis method.

Liao [15] details the HAM technique in solving nonlinear differential equations. 


\section{Mathematical formulation}

We consider a batch reactor operated isothermally with negligible volume change due to reaction and with two elementary reactions

$$
\mathrm{A}+\mathrm{B} \longrightarrow \mathrm{C} \text { and } \mathrm{C}+\mathrm{B} \longrightarrow \mathrm{D} \text {. }
$$

Examples of chemical processes that are governed by mechanism (1) include potassium permanganate $(\mathrm{A})+$ ethanol $(\mathrm{B}) \rightarrow$ acetic acid $(\mathrm{C})$, and then

$$
\text { acetic } \operatorname{acid}(\mathrm{C})+\text { ethanol }(\mathrm{B}) \rightarrow \text { ethyl acetate }(\mathrm{D}),
$$

and also the nitric acid oxidation of 2 octanol to 2 octanone and further oxidation of 2 octanone to carboxylic acids. Application of the material balance for the constant volume reactor gives the following differential equations

$$
\begin{aligned}
\frac{d[A]}{d t} & =-k_{1}[A][B], \\
\frac{d[B]}{d t} & =-k_{1}[A][B]-k_{2}[C][B], \\
\frac{d[C]}{d t} & =k_{1}[A][B]-k_{2}[C][B], \\
\frac{d[D]}{d t} & =k_{2}[C][B],
\end{aligned}
$$

where $k_{1}$ and $k_{2}$ are reaction rate constants, and where [.] denotes concentration. The initial concentrations are

$$
[A](0)=A_{0}, \quad[B](0)=B_{0}, \quad[C](0)=0, \quad[D](0)=0 .
$$

For brevity, we introduce the following notation to denote the concentrations of the reactants and products

$$
y_{1}(t)=[A](t), \quad y_{2}(t)=[B](t), \quad y_{3}(t)=[C](t), \quad y_{4}(t)=[D](t)
$$


Equation (7) is uncoupled from system (4)-(6), thus the solution for [D](t) can obtained by integration of $(7)$ when the solutions for $[B](t)$ and $[C](t)$ are known. Using the notation (9), equations (4)-(6) transform to

$$
\begin{aligned}
& \frac{d y_{1}(t)}{d t}=-k_{1} y_{1}(t) y_{2}(t), \\
& \frac{d y_{2}(t)}{d t}=-k_{1} y_{1}(t) y_{2}(t)-k_{2} y_{3}(t) y_{2}(t), \\
& \frac{d y_{3}(t)}{d t}=k_{1} y_{1}(t) y_{2}(t)-k_{2} y_{3}(t) y_{2}(t) .
\end{aligned}
$$

The initial conditions become

$$
y_{1}(0)=A_{0}, \quad y_{2}(0)=B_{0}, \quad y_{3}(0)=0 .
$$

In the next section equations (10)-(13) are solved using the homotopy analysis method (HAM).

\section{Homotopy analysis method solution}

The concentrations for the reacting species $\mathrm{A}, \mathrm{B}$ and $\mathrm{C}$ all tend to zero as $t \rightarrow \infty$. We therefore assume that

$$
y_{1}(t)=\sum_{j=1}^{+\infty} a_{j} e^{-j \beta t}, \quad y_{2}(t)=\sum_{j=1}^{+\infty} b_{j} e^{-j \beta t}, \quad y_{3}(t)=\sum_{j=1}^{+\infty} c_{j} e^{-j \beta t},
$$

where $a_{j}, b_{j}$ and $c_{j}$ are coefficients. The parameter $\beta$ is a convergence controlling auxiliary parameter that is characteristic of the HAM approach and is carefully selected in such a way that the resulting HAM solution conforms to the rule of solution expression. These expressions provide us with the socalled rule of solution expressions for solving the governing equations (10)(13). The $\beta$ value is experimentally selected through trial and error for a fixed value of the HAM auxiliary parameter $\hbar$. However, a fixed value of $\hbar$ 
in most cases will work for a reasonable non-zero values of $\beta$ and still gives similar agreement between the numerical solution and the HAM solution.

To obtain solutions in the form of (14), we use the HAM auxiliary linear operators

$$
\mathcal{L}_{i}\left[\Phi_{i}(t ; q)\right]=\frac{\partial \Phi_{i}(t ; q)}{\partial t}+\beta \Phi_{i}(t ; q)
$$

which have the properties

$$
\mathcal{L}_{i}\left[C_{i} e^{-\beta t}\right]=0,
$$

where $C_{i}(i=1,2,3)$ are integral coefficients, $q \in[0,1]$ is the HAM embedding parameter, $\Phi_{i}(\mathrm{t} ; \mathbf{q})$ are unknown functions. The governing equations (10)(12) suggest that we define the following HAM nonlinear operators:

$$
\begin{aligned}
& \mathcal{N}_{1}\left[\Phi_{i}(\mathrm{t} ; \mathrm{q})\right]=\frac{\partial \Phi_{1}(\mathrm{t} ; \mathrm{q})}{\partial \mathrm{t}}+\mathrm{k}_{1} \Phi_{1}(\mathrm{t} ; \mathrm{q}) \Phi_{2}(\mathrm{t} ; \mathrm{q}) \\
& \mathcal{N}_{2}\left[\Phi_{\mathrm{i}}(\mathrm{t} ; \mathrm{q})\right]=\frac{\partial \Phi_{2}(\mathrm{t} ; \mathrm{q})}{\partial \mathrm{t}}+\mathrm{k}_{1} \Phi_{1}(\mathrm{t} ; \mathrm{q}) \Phi_{2}(\mathrm{t} ; \mathrm{q})+\mathrm{k}_{2} \Phi_{2}(\mathrm{t} ; \mathrm{q}) \Phi_{3}(\mathrm{t} ; \mathrm{q}) \\
& \mathcal{N}_{3}\left[\Phi_{i}(\mathrm{t} ; \mathrm{q})\right]=\frac{\partial \Phi_{3}(\mathrm{t} ; \mathrm{q})}{\partial \mathrm{t}}-\mathrm{k}_{1} \Phi_{1}(\mathrm{t} ; \mathrm{q}) \Phi_{2}(\mathrm{t} ; \mathrm{q})+\mathrm{k}_{2} \Phi_{2}(\mathrm{t} ; \mathrm{q}) \Phi_{3}(\mathrm{t} ; \mathrm{q})
\end{aligned}
$$

Let $\hbar$ be an auxiliary parameter and $\mathrm{H}(\mathrm{t})$ be an auxiliary function. Using the embedding parameter $\mathrm{q}$ we construct the so called zeroth order deformation equations as

$$
(1-q) \mathcal{L}_{i}\left[\Phi_{i}(t ; q)-y_{i, 0}(t)\right]=\hbar q H(t) \mathcal{N}_{i}\left[\Phi_{i}(t ; q)\right]
$$

subject to the conditions $\Phi_{i}(t, 0)=y_{i, 0}(t)$, where $y_{i, 0}(t)$ are the initial guesses of $y_{i}(t)$. Considering the initial conditions (13) and the expressions (14) we choose the initial guesses

$$
y_{1,0}(t)=A_{0} e^{-\beta t}, \quad y_{2,0}(t)=B_{0} e^{-\beta t}, \quad y_{3,0}(t)=0 .
$$

When $\mathrm{q}=0$ and $\mathrm{q}=1$ we have

$$
\Phi_{i}(t ; 0)=y_{i, 0}(t), \quad \Phi_{i}(t ; 1)=y_{i}(t) .
$$


Thus, as $\mathrm{q}$ varies from 0 to 1 , the solutions $\Phi_{\mathfrak{i}}(\mathrm{t} ; \mathrm{q})$ vary from the initial guesses $y_{i, 0}(t)$ to the exact solutions $y_{i}(t)$. Expanding $\Phi_{i}(t ; q)$ using Taylor series with respect to $\mathrm{q}$, we obtain,

$$
\Phi_{i}(t ; q)=\Phi_{i}(t ; 0)+\sum_{m=1}^{+\infty} y_{i, m}(t) q^{m}
$$

where

$$
y_{i, m}(t)=\left.\frac{1}{m !} \frac{\partial^{m} \Phi_{i}}{\partial q^{m}}\right|_{q=0} .
$$

Importantly, the HAM approach gives us freedom to choose the auxiliary parameter $\hbar$ and function $\mathrm{H}(\mathrm{t})$. Assuming that $\hbar$ and $\mathrm{H}(\mathrm{t})$ are carefully selected so that series (23) converges at $\mathbf{q}=1$, we have

$$
y_{i}(t)=y_{i, 0}(t)+\sum_{m=1}^{+\infty} y_{i, m}(t) .
$$

The auxiliary function $\mathrm{H}(\mathrm{t})$ must be chosen in such a way that the resulting solutions conform to the rule of solution expression (14).

To obtain the so called higher order deformation equations, we define the vectors

$$
\vec{y}_{i, n}=\left(y_{i, 0}(t), y_{i, 1}(t), y_{i, 2}(t), \ldots, y_{i, n}(t)\right) .
$$

Differentiating (20) $\mathrm{m}$ times with respect to $\mathrm{q}$, then setting $\mathrm{q}=0$, and finally dividing the resulting equations by $m$ !, we obtain the $m$ th order deformation equations

$$
\mathcal{L}_{i}\left[y_{i, m}(t)-x_{m} y_{i, m-1}(t)\right]=\hbar H(t) R_{i, m}\left(\vec{y}_{i, m-1}(t)\right),
$$

subject to the initial conditions

$$
y_{i, m}(0)=0
$$


where

$$
\begin{aligned}
& R_{1, m}=y_{1, m-1}^{\prime}+k_{1} \sum_{j=0}^{m-1} y_{1, j} y_{2, m-1-j}, \\
& R_{2, m}=y_{2, m-1}^{\prime}+k_{1} \sum_{j=0}^{m-1} y_{1, j} y_{2, m-1-j}+k_{2} \sum_{j=0}^{m-1} y_{2, j} y_{3, m-1-j}, \\
& R_{3, m}=y_{3, m-1}^{\prime}-k_{1} \sum_{j=0}^{m-1} y_{1, j} y_{2, m-1-j}+k_{2} \sum_{j=0}^{m-1} y_{2, j} y_{3, m-1-j} .
\end{aligned}
$$

and

$$
x_{m}= \begin{cases}0, & m \leqslant 1 \\ 1, & m>1\end{cases}
$$

Equations (27)-(32) , form a system of uncoupled first order differential equations. Since the HAM approach allows us to choose the convergencecontrol auxiliary function $\mathrm{H}(\mathrm{t})$ and parameter $\hbar$, we set $\mathrm{H}(\mathrm{t})=e^{-\beta \mathrm{t}}$. This choice of $\mathrm{H}(\mathrm{t})$ ensures that the resulting approximate solutions for $y_{i}(t)$ do not violate the rule of solution (14). Setting $m=1$, substituting the initial guesses $y_{i, 0}(t)$ in $(27)-(32)$ gives

$$
\begin{aligned}
y_{1,1}^{\prime}+\beta y_{1,1} & =-\hbar A_{0}\left[\beta e^{-2 \beta t}+B_{0} k_{1} e^{-3 \beta t}\right], \\
y_{2,1}^{\prime}+\beta y_{2,1} & =-\hbar B_{0}\left[\beta e^{-2 \beta t}+A_{0} k_{1} e^{-3 \beta t}\right], \\
y_{3,1}^{\prime}+\beta y_{3,1} & =-\hbar A_{0} B_{0} k_{1} e^{-3 \beta t} .
\end{aligned}
$$

Solving the above equations subject to the initial conditions $y_{i, m}(0)=0$ gives

$$
\begin{aligned}
& y_{1,1}(t)=\frac{A_{0} \hbar}{2 \beta}\left[B_{0} k_{1} e^{-3 \beta t}+2 \beta e^{-2 \beta t}-\left(2 \beta+B_{0} k_{1}\right) e^{-\beta t}\right] \\
& y_{2,1}(t)=\frac{B_{0} \hbar}{2 \beta}\left[A_{0} k_{1} e^{-3 \beta t}+2 \beta e^{-2 \beta t}-\left(2 \beta+A_{0} k_{1}\right) e^{-\beta t}\right] \\
& y_{3,1}(t)=\frac{A_{0} B_{0} k_{1} \hbar}{2 \beta}\left[e^{-3 \beta t}-e^{-\beta t}\right] .
\end{aligned}
$$


The solutions for $y_{i, m}(t)(m \geqslant 2)$ can easily be found in a similar manner, especially using symbolic computation software such as Maple, Mathematica, MATLAB and others.

\subsection{Explicit series solution of the batch reaction kinetics equations}

By considering the first few solutions for $y_{i, m}(t)$ we obtain

$$
y_{1, m}=\sum_{j=1}^{2 m+1} a_{m, j} e^{-\beta j t}, \quad y_{2, m}=\sum_{j=1}^{2 m+1} b_{m, j} e^{-\beta j t}, \quad y_{3, m}=\sum_{j=1}^{2 m+1} c_{m, j} e^{-\beta j t}
$$

where $a_{m, j}, b_{m, j}$ and $c_{m, j}$ are coefficients. Substituting the series (39) into equations (27)-(32) we obtain the following recurrence formulas, for $2 \leqslant j \leqslant$ $2 \mathrm{~m}+1$

$$
\begin{aligned}
a_{m, j}= & \chi_{m} \chi_{2 m-j+1} a_{m-1, j}+\hbar \chi_{2 m-j+4} a_{m-1, j-1}-\frac{\hbar k_{1} \chi_{j-1} \lambda_{m, j-1}}{\beta(j-1)} \\
b_{m, j}= & \chi_{m} \chi_{2 m-j+1} b_{m-1, j}+\hbar \chi_{2 m-j+4} b_{m-1, j-1} \\
& -\frac{\hbar \chi_{j-1}\left(k_{1} \lambda_{m, j-1}+k_{2} \gamma_{m, j-1}\right)}{\beta(j-1)} \\
c_{m, j}= & \chi_{m} \chi_{2 m-j+1} c_{m-1, j}+\hbar \chi_{2 m-j+4} c_{m-1, j-1} \\
& -\frac{\hbar \chi_{j-1}\left(k_{2} \gamma_{m, j-1}-k_{1} \lambda_{m, j-1}\right)}{\beta(j-1)}
\end{aligned}
$$

where

$$
\lambda_{m, j}=\sum_{j=0}^{m-1} \sum_{r=\max \{1, i+2 j-2 n+1\}}^{\min \{i-1,2 j+1\}} a_{j, r} b_{m-1-j, i-r}
$$

and

$$
\gamma_{m, j}=\sum_{j=0}^{m-1} \sum_{r=\max \{1, i+2 j-2 n+1\}}^{\min \{i-1,2 j+1\}} b_{j, r} c_{m-1-j, i-r} .
$$


From the initial conditions $y_{i, m}(0)=0$, we obtain

$$
a_{0,1}=A_{0}, \quad b_{0,1}=B_{0}, \quad \text { and } \quad c_{0,1}=0 .
$$

Also,

$$
a_{m, 1}=-\sum_{j=2}^{2 m+1} a_{m, j}, \quad b_{m, 1}=-\sum_{j=2}^{2 m+1} b_{m, j}, \quad c_{m, 1}=-\sum_{j=2}^{2 m+1} c_{m, j} .
$$

Using the above recurrence relations, we obtain, in succession, all the coefficients starting from the coefficients of the initial guesses (45) and the solutions (36)-(38). This process results in the explicit series solution

$$
\begin{aligned}
& y_{1}(t)=\sum_{m=1}^{+\infty} \sum_{j=1}^{2 m+1} a_{m, j} e^{-j \beta t} \\
& y_{2}(t)=\sum_{m=1}^{+\infty} \sum_{j=1}^{2 m+1} b_{m, j} e^{-j \beta t} \\
& y_{3}(t)=\sum_{m=1}^{+\infty} \sum_{j=1}^{2 m+1} c_{m, j} e^{-j \beta t}
\end{aligned}
$$

The mth order approximation is

$$
\begin{aligned}
& y_{1}(t) \approx \sum_{m=1}^{M} \sum_{j=1}^{2 m+1} a_{m, j} e^{-j \beta t} \\
& y_{2}(t) \approx \sum_{m=1}^{M} \sum_{j=1}^{2 m+1} b_{m, j} e^{-j \beta t} \\
& y_{3}(t) \approx \sum_{m=1}^{M} \sum_{j=1}^{2 m+1} c_{m, j} e^{-j \beta t}
\end{aligned}
$$




\section{Results and discussion}

We present the HAM approach results and compare them to numerical methods of solution. We used Maple to obtain successive solutions of $A(t), B(t)$, $C(t)$ and $D(t)$ for $m \geqslant 1$. When applying the HAM technique, the auxiliary parameter $\hbar$ is critical in determining the convergence of the series. The convergence rate and region of the HAM solution series depends on the careful selection of the auxiliary parameter $\hbar$. As pointed out by Liao [15], the admissible values of $\hbar$ are chosen from the so called $\hbar$-curve in which some derivative property of the governing function, say $y_{1}^{\prime}(0)$ in the case of the current problem, is considered to be an independent variable and plotted against $\hbar$. The valid region of $\hbar$ where the series converges is the horizontal segment of each $\hbar$-curve. This study found that $\hbar=-1 / 4$ was appropriate to be used. Using this choice of the auxiliary parameter the HAM approximation at order $m=15$ was found to converge to the numerical solution. Figures 1-4 show comparisons of the HAM analytical solutions with numerical results obtained using MATLAB initial value solvers. As can be clearly observed in the figures, there is an excellent agreement between the two approaches for all values of time $t$. As expected, in Figure 1 we observe that as the reaction rate $k_{1}$ increases, concentration $A(t)$ which is a reactant is greatly reduced. It quickly diminishes for bigger values of the reaction rate. In Figure 2 we observe that the concentration profiles of reactant $B(t)$ are reduced as values of $k_{1}$ increase. The reduction of $B(t)$ is not as great as that of $A(t)$ since reactant $B(t)$ is also added to react with reactant $C(t)$ at the next phase of the batch reaction process. As expected, we observe in Figure 3 that concentration $C(t)$ increases as the reaction rate $k_{1}$ increases and reaches a peak before steadily decreases as it then reacts with $B(t)$ to produce concentrate $\mathrm{D}(\mathrm{t})$. The optimal time at which the concentration of the product $\mathrm{C}(\mathrm{t})$ is maximum can be deduced from Figure 3 and then the reactor quenched at this time. In Figure 4 we observe that as the reaction rate $k_{1}$ increases, the product concentration $\mathrm{D}(\mathrm{t})$ increases as expected. 


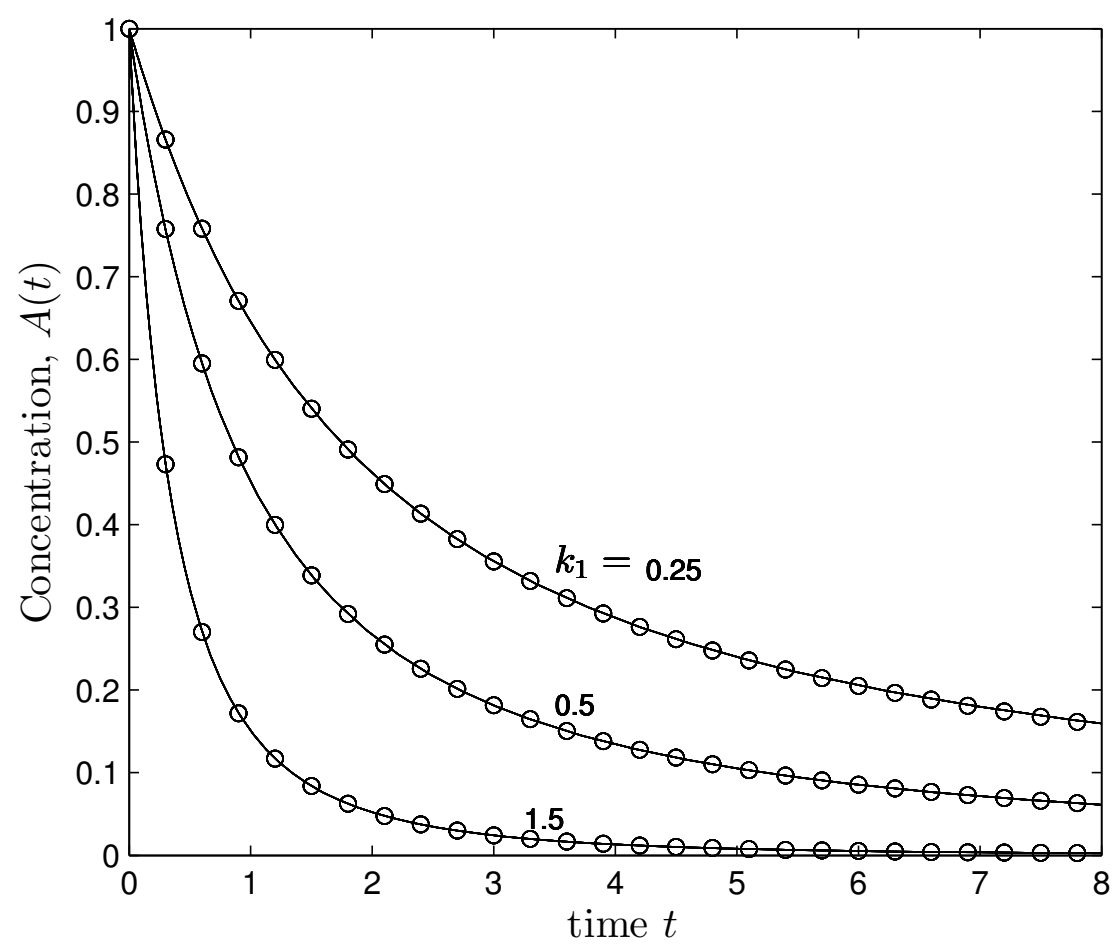

Figure 1: Comparison of the numerical solution of $A(t)$ with the 15 th order HAM approximate solution when $\hbar=-0.25, k_{2}=0.5, \beta=0.15$. The solid line denotes the numerical solution and the circles denote the HAM solutions 


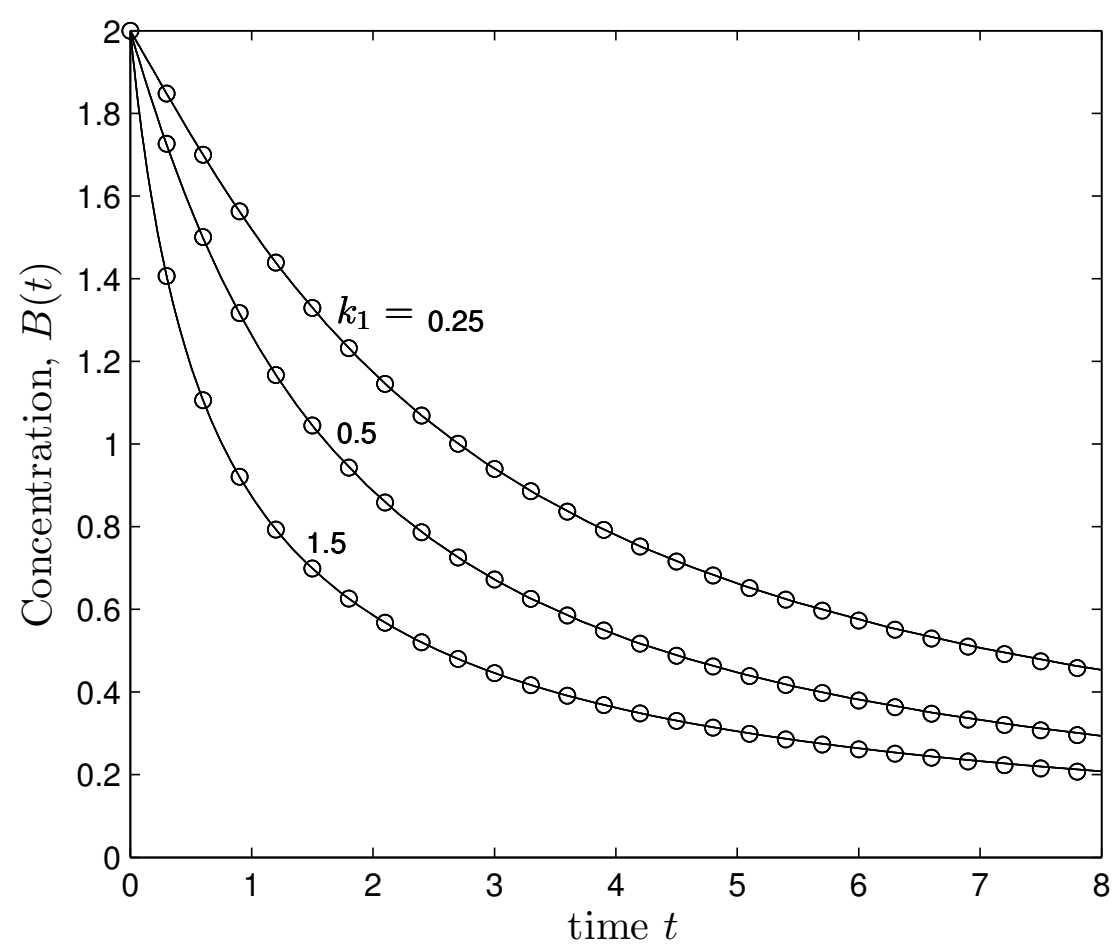

Figure 2: Comparison of the numerical solution of $\mathrm{B}(\mathrm{t})$ with the 15th order HAM approximate solution when $\hbar=-0.25, k_{2}=0.5, \beta=0.15$. The solid line denotes the numerical solution and the circles denote the HAM solutions 


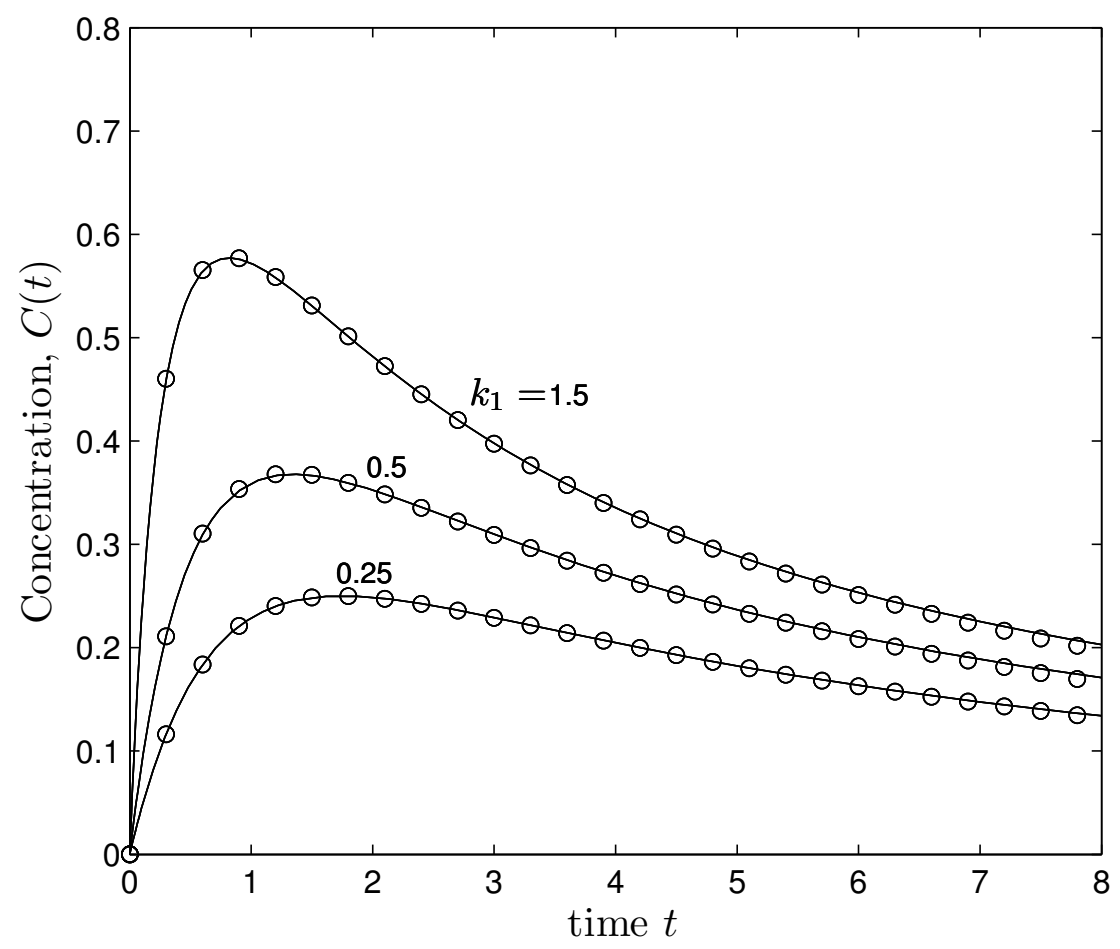

Figure 3: Comparison of the numerical solution of $\mathrm{C}(\mathrm{t})$ with the 15 th order HAM approximate solution when $\hbar=-0.25, k_{2}=0.5, \beta=0.15$. The solid line denotes the numerical solution and the circles denote the HAM solutions 


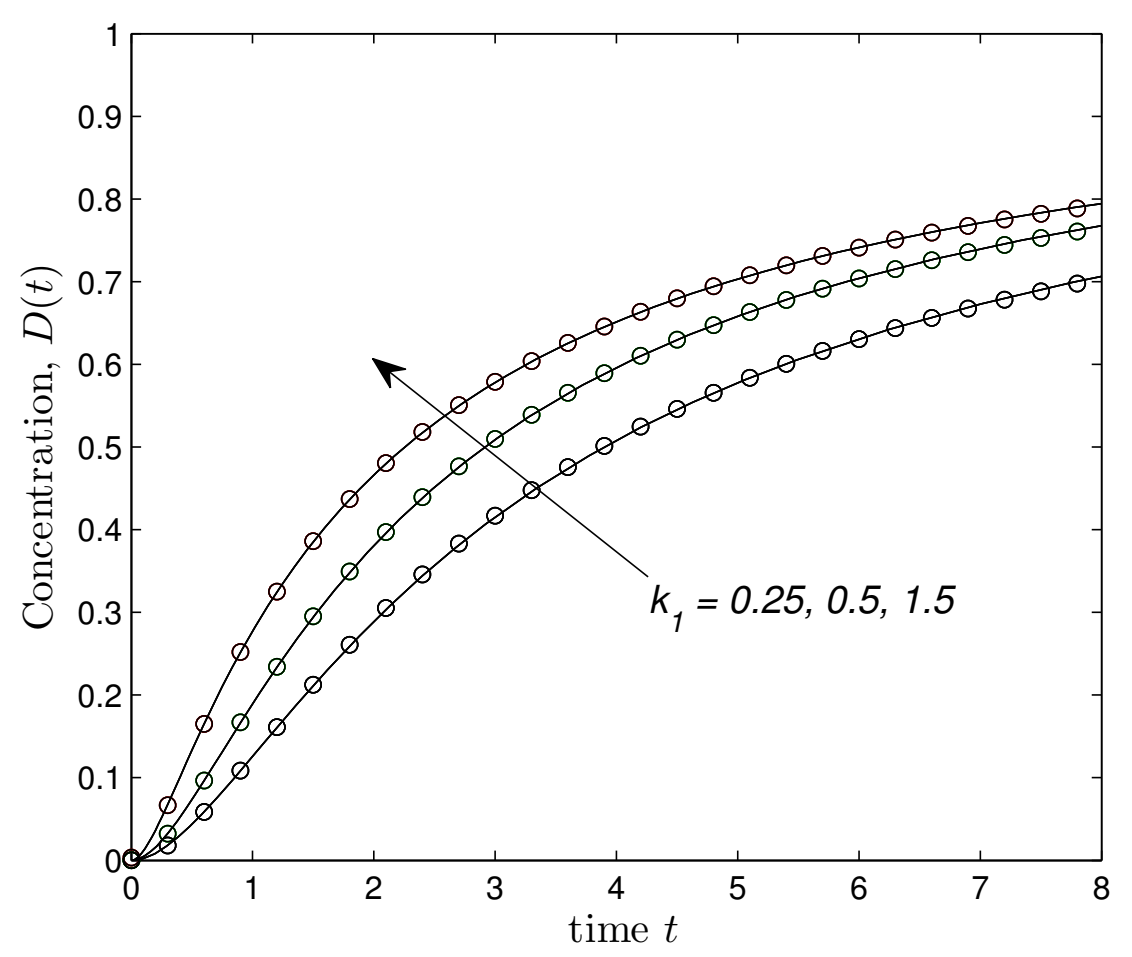

Figure 4: Comparison of the numerical solution of $D(t)$ with the 15th order HAM approximate solution when $\hbar=-0.25, k_{2}=0.5, \beta=0.15$. The solid line denotes the numerical solution and the circles denote the HAM solutions 


\section{Conclusion}

The homotopy analysis method is used to solve the nonlinear batch reaction kinetic equations. Explicit series solutions describing the time evolution of the underlying reaction kinetics equations are obtained. Our HAM results are found to be in excellent agreement with numerical results. This confirms the power and significance of the HAM approach as an effective technique for solving nonlinear systems of equations. The value of 0.15 for $\beta$ was arrived at when the appropriate value of $\hbar=-1 / 4$ was used. We hope that the HAM approach presented here will spawn further interest in the analysis of reaction kinetic models that fully characterize the reactions without making assumptions of small or large concentration, initial or long term effects, quasisteady or steady state effects.

Acknowledgements The authors are most grateful to the anonymous reviewers for their helpful comments, and to P. E. Mojapelo for his priceless technical assistance.

\section{References}

[1] D. W. T. Rippin, Simulation of single andmultiprod uct batch chemical plants for optimal design and operation, Computers and Chemical Engineering, 7, (3), 1983, 137-156. doi:10.1016/0098-1354(83)85016-9 E38

[2] M. Friedrich and R. Perne, Design and control of batch reactors an industrial viewpoint, Computers and Chemical Engineering, 19s, 1995, S357-S368. doi:10.1016/0098-1354(95)87063-6 E38 
[3] Y. Kawarasai, T. Kawai, H. Nakano and T. Yamane, A long-lived batch reaction system of a cell-free protein synthesis, Analytical, Biochemistry, 226, 1995, 320-324. E38

[4] M. Fulcher, J. Warzywoda, A. Sacco, Jr., R. W. Thompson, and A. G. Dixon, Gel shrinkage and nutrient addition in unstirred batch zeolite A reaction systems, Microporous Materials, 10, 1997, 199-209. doi:10.1016/S0927-6513(97)00008-4 E38

[5] D. Bonvin, Optical operation of batch reactors-personal view, Published by Elsevier Science Ltd, 1998. E38

[6] B. A. A. van Woezik and K. R. Westerterp, Runaway behavior and thermally safe operation of multiple liquid reactions in the semi-batch reactor The nitric acid oxidation of 2-octanol, Chemical Engineering and Processing, 41, 2001, 59-77. E39

[7] K. R. Muske, M. Badlani, P. C. Dell'Orco, J. Brum, Open-loop optimization for batch reaction systems: a case study, Chemical Engineering Science, 59, 2004, 1167-1180. doi:10.1016/j.ces.2003.12.016 E39

[8] X. Hua., S. Rohani, and A. Jutan, Cascade closed-loop optimization and control of batch reactors, Chemical Engineering Science, 59, 2004, 5695-5708. doi:10.1016/j.ces.2004.04.002 E39

[9] J. Zhang, and R. Smith, Design andoptimisation of batch andsemi-batch reactors, Chemical Engineering Science, 59, 2004, 459-478. E39

[10] L. R. B. Goncalves, R. L. C. Giordano, R. C. Giordano, Mathematical modeling of batch and semibatch reactors for the enzymic synthesis of amoxicillin, Process Biochemistry, 40, 2005, 247-256.

doi:10.1016/j.procbio.2003.12.010 E39 
[11] A. K. Jana., and P. V. R. Adari, Nonlinear state estimation and control of a batch reactive distillation, Chemical Engineering Journal, 150, 2009, 516-526. doi:10.1016/j.cej.2009.03.015 E39

[12] S. J. Liao, An explicit, totally analytic approximation of Blasius viscous flow problems, Int. J. Non-Linear Mech, 34, 4, 1999, 759-778. E39

[13] S. J, Liao, On the homotopy analysis method for nonlinear problems, Appl Math Comput, 147, 2004, 499-513. doi:10.1016/S0096-3003(02)00790-7 E39

[14] S. J. Liao, Notes on the homotopy analysis method: Some defintions and theorems, Communications in Nonlinear Science and Numerical Simulation, 14, 2009, 983-997. doi:10.1016/j.cnsns.2008.04.013 E39

[15] S. J. Liao, Beyond Perturbation: Introduction to Homotopy Analysis Method. Chapman \& Hall/CRC Press, 2003. E40, E48

[16] A. Mehmood and A. Ali, Analytic solution of generalized three dimensional flow and heat transfer over a stretching plane wall, International Communications in Heat and Mass Transfer, 33, 2006, 1243-1252. doi:10.1016/j.icheatmasstransfer.2006.08.001 E39

[17] A. Ali and A. Mehmood, Homotopy analysis of unsteady boundary layer flow adjacent to permeable stretching surface in a porous medium, Communications in Nonlinear Science and Numerical Simulation, 13, 2008, 340-349. doi:10.1016/j.cnsns.2006.03.008 E40

[18] A. Mehmood, A. Ali and T. Shah, Heat transfer analysis of unsteady boundary layer flow by homotopy analysis method, Communications in Nonlinear Science and Numerical Simulation, 13, 2008, 902-912. doi:10.1016/j.cnsns.2006.09.008 E40

[19] T. Hayat, M. Sajid and I. Pop, Three-dimensional flow over a stretching surface in a viscoelastic fluid, Nonlinear Analysis: Real 
World Applications, 9, 2008, 1811-1822.

doi:10.1016/j.nonrwa.2007.05.010 E40

[20] M. Sajid and T. Hayat, Comparison between HAM and HPM solutions in heat radiation equations, International communications in Heat and Mass Transfer, 36, 2009, 59-62.

doi:10.1016/j.icheatmasstransfer.2008.08.010 E40

[21] J. Cheng, S. J. Liao, R. N. Mohapatra and K. Vajravelu, Series solutions of nano boundary layer flows by means of the homotopy analysis method, J. Math. Anal. Appl., 343, 2008, 233-245. doi:10.1016/j.jmaa.2008.01.050 E40

[22] A. Alizadeh-Pahlavan, and K. Sadeghy, On the use of homotopy analysis method for solving unsteady MHD flow of Maxwelian fluids above impulively stretching sheets, Communications in Nonlinear Science and Numerical Simulation, 14, 2009, 1355-1365. E40

[23] H. Bararnia, A. Ghotbi and G. Domairry, On the analytic solution for MHD natural convection flow and heat generation fluid in porous medium, Communications in Nonlinear Science and Numerical Simulation, 14, 2009, 2689-2701. doi:10.1016/j.cnsns.2008.09.018 E40

[24] H. Khan, S. Liao, R. N. Mohapatra and K. Vajravelu, An analytical solution for a nonlinear time-delay model in biolgy, Nonlinear Sci Numer Simul, 14, 2009, 3141-3148. doi:10.1016/j.cnsns.2008.11.003 E40

[25] F. M. Allan: Construction of analytic solution to chaotic dynamical systems using Homotopy analysis method, Chaos, Solutions and Fractals, 39, 2009, 1744-1752. doi:10.1016/j.chaos.2007.06.116 E40

[26] H. Xu, S. Liao and X. You, Analysis of nonlinear fractional partial differential equations with the homotopy analysis method, Nonlinear Sci Numer Simul, 14, 2009, 1152-1156.

doi:10.1016/j.cnsns.2008.04.008 E40 


\section{Author addresses}

1. S. S. Motsa, Department of Mathematics, University of Swaziland, Private Bag 4, Kwaluseni, SwaziLAnd.

mailto: sandile@science.uniswa.sz

2. S. Shateyi, University of Venda, Department of Mathematics, Private Bag X5050, Thohoyandou 0950, South AfRICA. mailto:stanford.shateyi@univen.ac.za 